\title{
The problem of high expressed emotion
}

Authors: Do Amaral, S.; Vian F; Pimenta S.

Resident Doctor of Childhood and Adolescense Psychiatry at Centro Hospitalar Universitário de Lisboa Central, Hospital Dona Estefânia.

Introduction: Expressed Emotion (EE) is defined by attitudes of high criticism, hostility, and/or emotional over-involvement of caregivers. High EE is a risk factor of interest in several forms of youth psychopathology, it has been linked to poorer clinical course, diminished treatment response, and higher rates of relapse for depressive, anxiety and bipolar disorders. It is one of the most robust predictors of long-term outcome in the adult psychopathology literature.

\begin{abstract}
Objective and Methods: This paper has the objective to study the impact on child and adolescent mental health associated with parental high expressed emotion. The methods used were a bibliographic review carried out through the research in PubMed database using the following keywords: "parental high expressed emotion", "high expressed emotion within families", "child and adolescent mental health", "approach on parental expressed emotion".
\end{abstract}

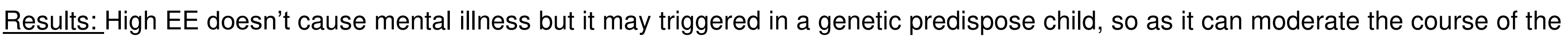
child illness.

EE has been associated with early temperamental abnormalities, self-injurious behavior, and higher levels of externalizing comorbidity. Studies among adolescents with eating disorders showed that maternal criticism accounted for 28-34 \% of the variance in treatment outcome. Parental EE also predicted treatment response and functional outcomes among youth receiving exposure-based treatment for obsessive compulsive disorder. Among adolescents with bipolar disorder, EE moderated the effects of psychosocial treatment, such that youth whose parents were high-EE at baseline showed greater improvement in depression and mania scores in familyfocused treatment compared to youth in a brief treatment control.

How EE attitudes interacts with the unfolding of childhood psychopathology is unknown, which undermine efforts to intervene effectively with maladaptive family dynamics. But some studies in bipolar disorder have postulate that excessive activation of the hypothalamicpituitary-adrenal (HPA) axis may reflect inadequate neuroendocrine control over the immune system, leading to increased levels of inflammatory markers and a more deleterious course of illness.

There are some studies who explain the "toxic family models", families who have the sustained maladaptive pattern of responding to stress with criticism, blame, or excessive control and may create emotionally charged environments that exacerbate symptoms, which in turn may fuel negative parent/child interactions and interfere with healthy behavior management strategies.

Many articles also demonstrate that high EE attitudes have been linked to elevated rates of psychopathology in parents, and is also associated with lower levels of perceived parental support, poorer family communication and higher levels of marital problems and family conflict. These findings suggest that EE is related to numerous aspects of family functioning that might undermine the ability of parents to cope effectively with child psychopathology.

Conclusion: Psychoeducation alone is unlikely to produce changes in affectively charged family dynamics. Despite research linking high EE to poor outcomes for mental illness, parallel research on effective interventions for EE are lacking. Most family therapy trials have been focused on reduction of symptoms as main goal. Research should focused on effective approach to high EE to more positive communication for more successful interventions. Studies of family interaction suggest the importance of training communication and problem-solving skills to reduce the frequency and intensity of negative exchanges or to increase the frequency of positive interactions. Research that examines the impact of critical or overinvolved home environments at the neural or immunological level may clarify the mechanisms by which EE leads to poorer outcomes of psychiatric disorder and help to identify novel strategies for intervention. 